\title{
Cycle biologique de Parampbistomum togolense n. sp. (Trematoda, Paramphistomidae)
}

par J.-L. ALBARET *, Christiane BAYSSADE-DUFOUR *, J. GUILHON **, S. D. KULO *** et H. PICOT ****

* Laboratoire de Zoologie (Vers) associé au C.N.R.S., Muséum national d'Histoire naturelle, 43, rue Cuvier, F 75231 Paris Cedex 05.

** Laboratoire de Parasitologie, Ecole Nationale Vétérinaire, 7, avenue du Général-de-Gaulle, F 94700 Maisons-Alfort.

*** Laboratoire de Parasitologie, Université du Bénin, B.P. 1515, Lomé, Togo.

***** Laboratoire de Parasitologie, Faculté de Médecine, Broussais-Hôtel-Dieu, 15, rue de l'Ecole-de-Médecine, F 75006 Paris.

\section{Résumé.}

Paramphistomum togolense $\mathrm{n}$. sp. a été obtenu sous sa forme adulte à partir d'un Mouton infesté expérimentalement par des métacercaires issues de cercaires émises par des Bulinus (Bulinus) forskalii naturellement parasités recueillis dans les environs immédiats de Lomé (Togo). Les miracidiums ont permis d'infester au laboratoire ce même Mollusque qui a fourni de nombreuses cercaires.

Les différents stades larvaires sont étudiés. Les caractères morphologiques et histologiques de l'adulte et la forme très particulière du sporocyste distinguent ce Trématode des autres espèces connues du genre.

Les formations argyrophiles superficielles du miracidium et de la cercaire sont décrites en détail car elles nous paraissent constituer les meilleurs caractères utilisables pour la taxonomie de ce groupe.

Accepté le 14 février 1978. 


\section{Sumimary.}

Life cycle of Paramphistomum togolense n. sp. (Trematoda, Paramphistomidae).

Mature worms were obtained from a sheep experimentally infested with metacercariae issued from cercariae emitted by naturally infested snails : Bulinus (Bulinus) forskalii collected near Lomé (Togo). Miracidia were used for infection of these laboratory-reared snails which gave numerous cercariae.

Larval stages are studied. Morphological and histological characters of the adult and the very peculiar shape of the sporocyst distinguish this trematode from the other known species of the genus. The superficial argentophilic structures of the miracidium and cercaria are described in detail because, to our point, they constitute the best criteria for the taxonomy of this group.

La détermination des Paramphistomes est essentiellement basée sur la morphologie et l'histologie des formes adultes. Or la stabilité des caractères et leur valeur relative prêtent à controverse et la systématique de ce groupe reste très difficile. Dans le but de fonder une taxonomie et une phylogénie plus cohérentes des Trématodes, certains auteurs se sont attachés à l'étude des formes larvaires et particulièrement à celle de la disposition des organites argyrophiles superficiels du miracidium et de la cercaire : papilles sensorielles, pores excréteurs, ouvertures des glandes. Dans le cas des Paramphistomes ces recherches sont encore très limitées. Si de rares observations concernent le miracidium, aucune description précise n'a, à notre connaissance, été faite dans ce domaine chez les cercaires. Ayant eu l'occasion de réaliser le cycle d'un Paramphistome de Mammifère du Togo, nous nous sommes efforcés de suivre les différents stades larvaires en mettant particulièrement l'accent sur les formations argyrophiles superficielles du miracidium et de la cercaire.

\section{Matériel et méthodes}

Les métacercaires sont issues de cercaires émises en grand nombre par des Mollusques Bulinus (Bulinus) forskalii (Ehrenberg) récoltés à $5 \mathrm{~km}$ au Nord de Lomé (Togo) dans une mare permanente où viennent s'abreuver les troupeaux de Bovins. Après cinq semaines de maturation à $4{ }^{\circ} \mathrm{C}$, les métacercaires au nombre de 300 sont ingérées par un Mouton sain, âgé de 6 mois, élevé à l'Ecole Vétérinaire de Maisons-Alfort. Les premiers œufs sont observés dans les fèces cinq mois et demi après l'infestation. Dans la panse du Mouton sacrifié sept mois après l'infestation 92 Paramphistomes adultes sont récoltés. Les œufs recueillis dans de l'eau d'Evian sont conservés à une température de $27^{\circ} \mathrm{C}$ à l'obscurité. Après éclosion les miracidiums sont mis en présence de Bulinus (Bulinus) forskalii sains provenant de la Faculté de Médecine de Paris (souche importée du Sénégal en 1970), élevés à $23^{\circ} \mathrm{C}$, et nourris de laitue cuite. Le début de l'émission des cercaires a lieu 26 jours après 
l'infestation des Mollusques. Des métacercaires obtenues sont ingérées par un second Mouton sain. Ce dernier ayant dû être abattu 233 jours après l'infestation, 118 Paramphistomes adultes sont récoltés dans la panse.

Les adultes ont été étudiés en coupes frontales, sagittales et transversales colorées à l'hémalun de Masson et à l'hématoxyline de Regaud. Les stades larvaires ont fait
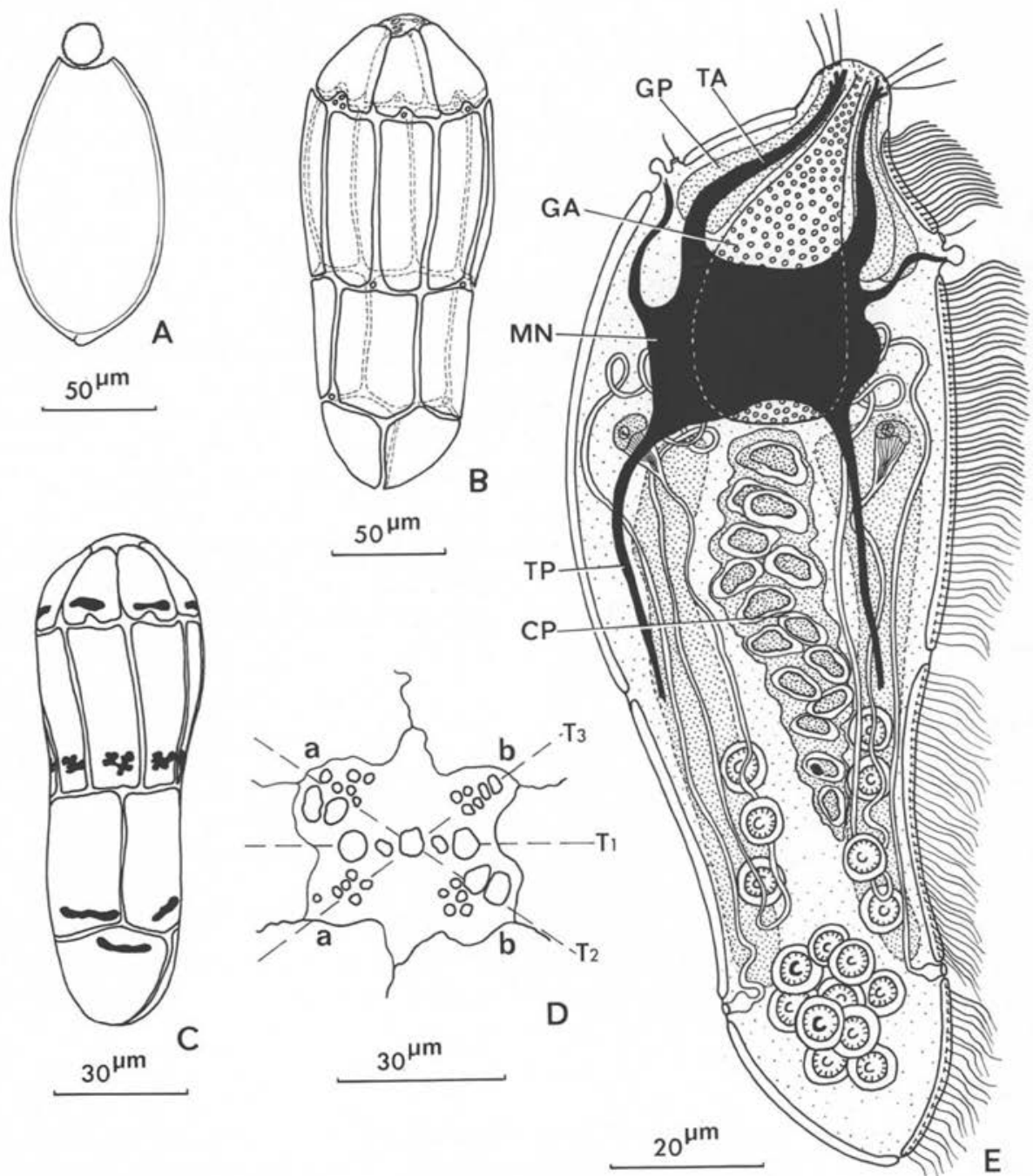

Fig. 1. A : E Euf après éclosion; B-E : Miracidium; B : Disposition des cellules ciliées et des organites argyrophiles superficiels; C : Noyaux des cellules ciliées; D : Vue apicale, disposition des organites argyrophiles sur le térébratorium; E : Morphologie générale, vue dorsale. 
l'objet d'observations «in vivo ». De plus les miracidiums ont été imprégnés à l'argent selon diverses techniques utilisant le nitrate d'argent : Méthode de Lynch (1933) avec montage dans la gomme au chloral de Faure, de Chatton et Lwoff (1930), réduction par l'hydroquinone ; et le protéinate d'argent : Méthode de Bodian modifiée par Dragesco et Njiné (1971). Les noyaux ont été mis en évidence par la réaction de Feulgen.

L'étude chétotaxique de la cercaire a pu être faite après imprégnation au nitrate d'argent selon la méthode de Combes, Bayssade-Dufour et Cassone (1976) et la méthode de Lynch.

\section{Observations}

\section{L'œuf.}

L'œuf, de couleur brunâtre, ovoïde, mesure $135 \mu \mathrm{m}$ de long et $70 \mu \mathrm{m}$ de large. Il possède un épaississement à son pôle postérieur. Lors de l'éclosion l'opercule, antérieur, d'un diamètre d'une vingtaine de microns se détache de la coque selon une ligne finement denticulée (fig. 1, A).

\section{Le miracidium.}

Les œufs éclosent à la lumière au cours des 9 à 12 jours suivant la ponte et libèrent des miracidiums qui nagent activement.

Le miracidium fixé en extension dans l'acide osmique à $2 \%$ mesure environ $200 \mu \mathrm{m}$ de long et $40 \mu \mathrm{m}$ de large.

\section{ANATOMIE EXterne.}

\section{Ciliature locomotrice :}

Les cils s'insèrent sur des cellules épidermiques au nombre de 20 réparties en quatre étages comprenant d'avant en arrière $6,8,4$ et 2 cellules séparées par trois ceintures : antérieure, moyenne et postérieure ( $f i g .1, B$ ).

Les noyaux des cellules ciliées épidermiques sont allongés transversalement en forme de batonnet ou plus ou moins ramifiés; ils se situent à la partie postérieure des cellules des trois premiers étages et à la partie antérieure des cellules du quatrième étage (fig. $1, C$ ).

\section{Organites superficiels.}

- Tubercules. Au niveau de la ceinture antérieure, diamétralement opposés, s'observent deux tubercules saillants sphériques, de $3 \mu \mathrm{m}$ de diamètre portés par un petit pédicule (fig. 1,E). Fréquemment observés chez les miracidiums de nombreux Trématodes et situés dans le même plan que les pores excréteurs ils définissent selon les auteurs le plan frontal du miracidium. 
- Organites argyrophiles. Au niveau du térébratorium se situent une trentaine de formations plus ou moins circulaires (fig. 1, D). Pour la commodité de la description nous utiliserons les axes de références de Swart (1967).

Selon l'axe T1 confondu avec le plan frontal de part et d'autre d'une formation centrale de $6 \mu \mathrm{m}$ de diamètre nous observons une formation d'un diamètre de $3 \mu \mathrm{m}$ et une autre plus latérale de $6 \mu \mathrm{m}$ de diamètre.

Selon l'axe T2 les groupes a et b comprennent chacun deux formations ovoïdes, contiguës de $6 \mu \mathrm{m}$ de longueur et cinq à sept formations plus petites d'un diamètre de $2 \mu \mathrm{m}$.

Selon l'axe T3 les groupes a et b sont constitués de 4 à 5 formations de 2 à $3 \mu \mathrm{m}$ de diamètre.

Certaines des petites formations sont pourvues d'une soie raide longue de 6 à $7 \mu \mathrm{m}$.

Au niveau de la ceinture antérieure, dans une échancrure au milieu de la base de chacune des cellules ciliées du premier étage, se situe une papille ciliée. En outre au voisinage de chacun des tubercules latéraux s'observent deux petites formations circulaires (fig. 1, B).

La ceinture moyenne présente deux petites formations symétriques par rapport au plan sagittal considérées comme ventrales par les auteurs (fig. 1, B).

Au niveau de la ceinture postérieure s'ouvrent, dans le plan frontal, les deux pores excréteurs, un de chaque côté du miracidium (fig. 1, B).

\section{ANATOMIE INTERNE :}

Au niveau du térébratorium débouchent la glande apicale longue d'une cinquantaine de microns et les glandes de pénétration latérales dont nous n'avons pu préciser le nombre (fig. 1, E).

La masse nerveuse centrale est adossée à la glande apicale et occupe selon les auteurs une position dorsale. Elle émet dans une direction antérieure des fibres nerveuses vers le térébratorium et la ceinture antérieure et deux troncs postérieurs dorsaux.

En arrière de la masse nerveuse centrale un massif cellulaire compact, de forme variable, le plus souvent allongé, regroupe une vingtaine de cellules à gros noyaux considérées comme les cellules primordiales du sporocyste.

Latéralement deux masses granuleuses allongées débutent sous la masse nerveuse centrale et s'étendent jusqu'au niveau des pores excréteurs.

En outre on observe des cellules arrondies réparties irrégulièrement mais plus nombreuses dans la moitié postérieure du miracidium.

L'appareil excréteur comporte deux cellules flammes, à grand axe longitudinal ou oblique, localisées à la limite du tiers antérieur du miracidium; le canal excréteur qui en part effectue un trajet selon une direction postérieure puis remonte vers la masse nerveuse et effectue un certain nombre de circonvolutions avant d'acquérir de nouveau une direction postérieure jusqu'au pore excréteur. 
'En vue de leur infestation les Mollusques Bulinus (B.) forskalii sont isolés dans des petits tubes de verre contenant environ 1 millilitre d'eau d'Evian. Dans chaque tube les miracidiums sont alors introduits. Seuls les Mollusques infestés par 1 à 4 miracidiums et dont la taille atteint ou dépasse $5,5 \mathrm{~mm}$ survivent. Des Mollusques de taille inférieure ou de taille supérieure mais infestés avec 10 à 20 miracidiums meurent en une quinzaine de jours.

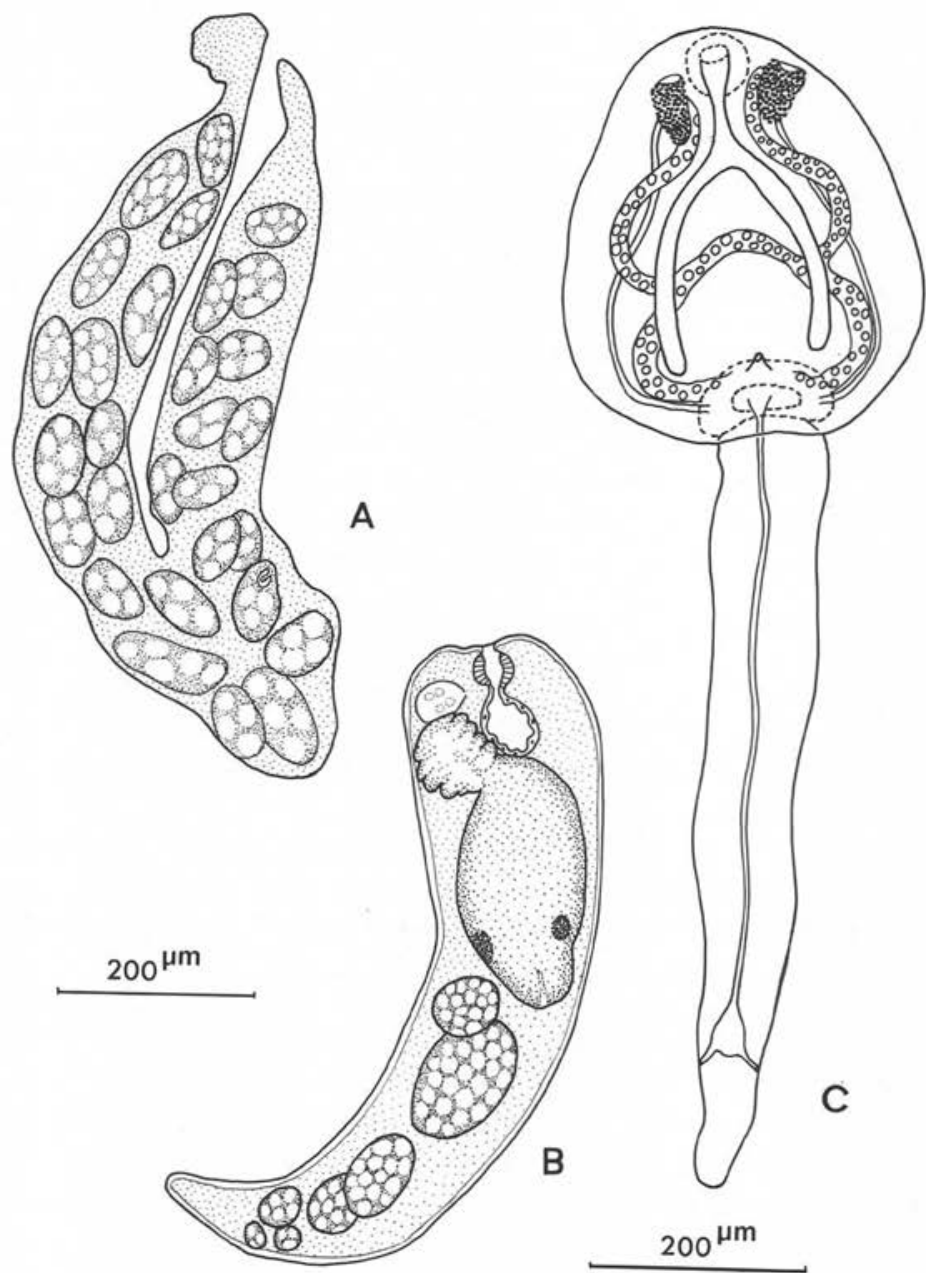

Fig. 2. A : Sporocyste âgé de 10 jours; B : Rédie âgée de 27 jours; C : Cercaire mûre, morphologie générale, vue dorsale.

\section{Le sporocyste.}

Les sporocystes observés 10 jours et 14 jours après l'infestation des Mollusques 
montrent une forme caractéristique en $\mathrm{V}$ très fermé (fig. 2, A). Leur taille augmente rapidement : $1400 \mu \mathrm{m} 10$ jours après l'infestation $2400 \mu \mathrm{m} 14$ jours après l'infestation. A ces stades le sporocyste renferme une trentaine de balles germinales encore iadifférenciées pour la plupart.

\section{La rédie.}

Les rédies observées 27 jours après l'infestation sont allongées et mesurent 390 à $770 \mu \mathrm{m}$ de long. Deux d'entre elles renferment une cercaire identifiable et 7 à 8 balles germinales (fig. 2, B).

Le pharynx sphérique de $40 \mu \mathrm{m}$ de diamètre environ débouche dans un sac digestif. La longueur de l'ensemble représente $1 / 5$ à $1 / 6$ de la longueur de la rédie.

\section{La cercaire.}

Dès le $19^{\circ}$ jour suivant l'infestation, des cercaires très immatures quittent la rédie et poursuivent leur maturation dans l'hépato-pancréas du Mollusque. Elles montrent au niveau où se formeront les ocelles des formations pigmentaires ramifiées.

Les premières cercaires mûres sont émises 26 jours après l'infestation du Mollusque. L'émission se poursuit pendant les semaines suivantes lorsque les Mollusques sont exposé à la lumière à une température de $27^{\circ} \mathrm{C}$. Il n'est pas possible d'obtenir plus d'une émission de cercaires par semaine.

La cercaire mûre est volumineuse (fig. 2, C) et son observation est rendue difficile par la présence de pigment et de cellules cystogènes à bâtonnets très abondants dans le corps cercarien qui mesure 400 à $450 \mu \mathrm{m}$ de long. La queue à peu près deux fois plus longue que le corps porte un voile hyalin, d'observation difficile, visible seulement « in vivo » et analogue à celui décrit par Lengy (1960) chez la cercaire de Paramphistomum microbothrium.

Les deux ocelles dorsaux longs de 50 à $60 \mu \mathrm{m}$, coniques, sont disposés longitudinalement dans la région antérieure du corps cercarien.

La ventouse orale a un diamètre externe de $50 \mu \mathrm{m}$. L'acétabulum postéro-ventral mesure $100 \mu \mathrm{m}$ de large.

L'œsophage est court et les caecums digestifs se terminent un peu avant le bord antérieur de l'acétabulum.

Etant donné l'opacité de la cercaire les cellules flammes n'ont pu être observées. La disposition des canaux excréteurs est analogue à celle des autres cercaires de Paramphistominae, et on y observe également des granules réfringents sphériques nombreux atteignant $12 \mu \mathrm{m}$ de diamètre. De même un pore médian dorsal s'ouvre dans la région postérieure du corps cercarien et deux pores latéraux débouchent latéralement vers l'extrémité de la queue.

\section{ChÉtotaxie.}

I. Région céphalique par hémicorps (fig. 3). 
CI $=1$ CIL, 1 CID.

CII comporte cinq papilles disposées régulièrement en demi-cercle.

On observe parfois une papille supplémentaire.

CIII $=2$ CIII 1,4 à 6 CIII 2.

$\operatorname{CIV}=7$ à $10 \operatorname{CIV} 1,6$ à $7 \operatorname{CIV} 2,6$ à $7 \operatorname{CIV} 3,16$ à $24 \operatorname{CIV} 4$.

$\mathrm{CV}=4$ à $5 \mathrm{CV} 1,4$ à $5 \mathrm{CV} 2,2$ à $3 \mathrm{CV} 3,3 \mathrm{CV} 4$.

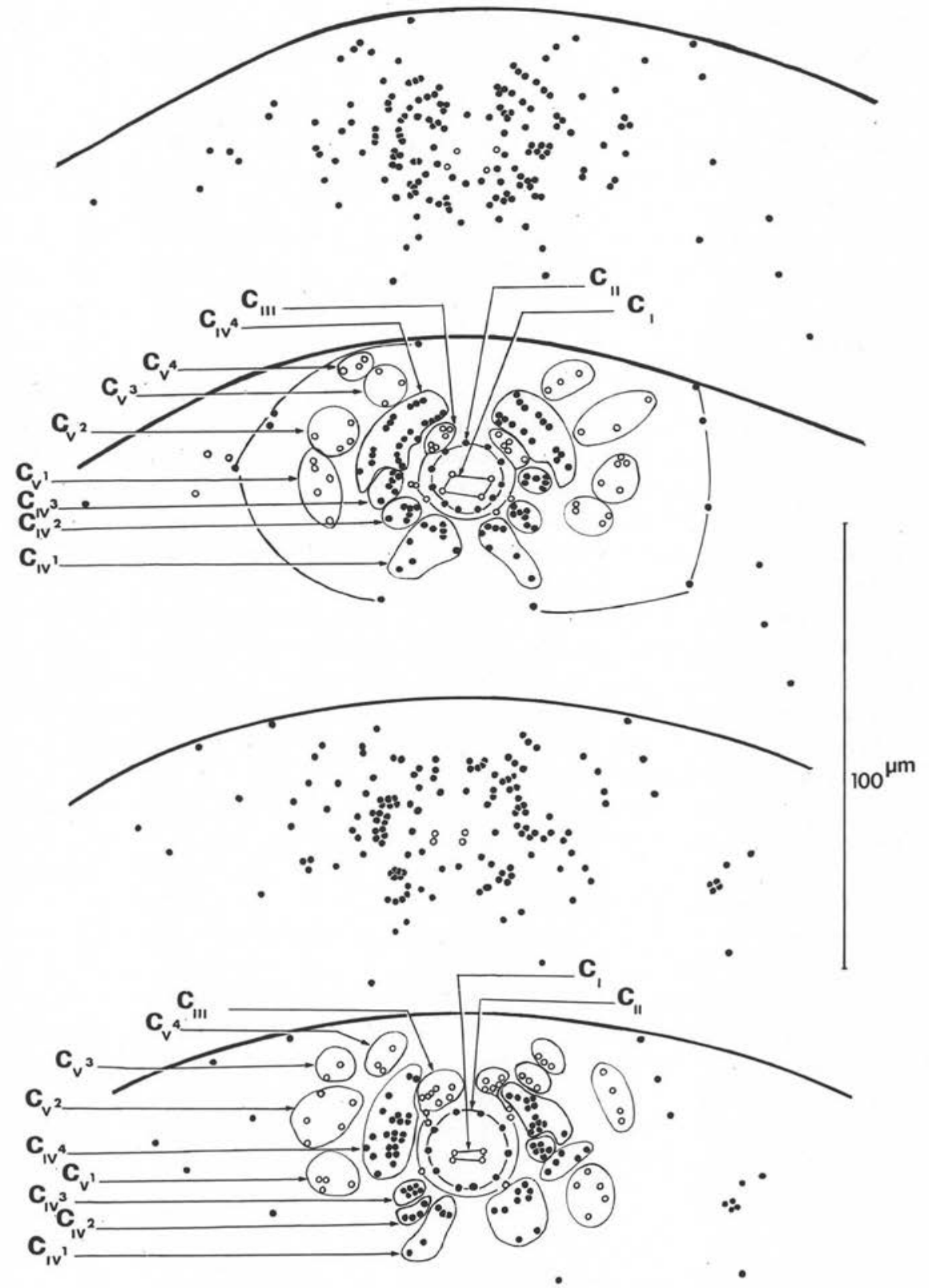

Fig. 3. Cercaire, chétotaxie de la région céphalique, vues ventrales. 
II. Région corporelle par hémicorps :

1. Papilles ventrales et dorsales (fig. $4, A$ et $B$ ).

$\mathrm{AI}=2$ AIV, 3 parfois 2 AID.

AII $=1$ AIIV, 2 AIID.

AIII $=2$ AIII V, 2 AIII D.

$\mathrm{M}=2 \mathrm{MV}, 1 \mathrm{MD}$.

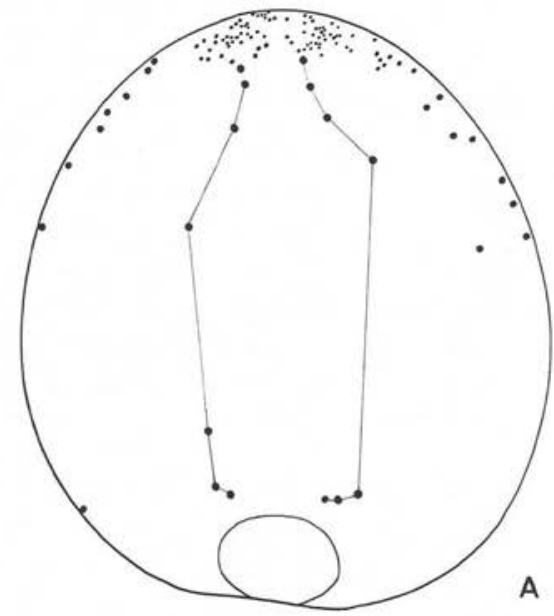

A
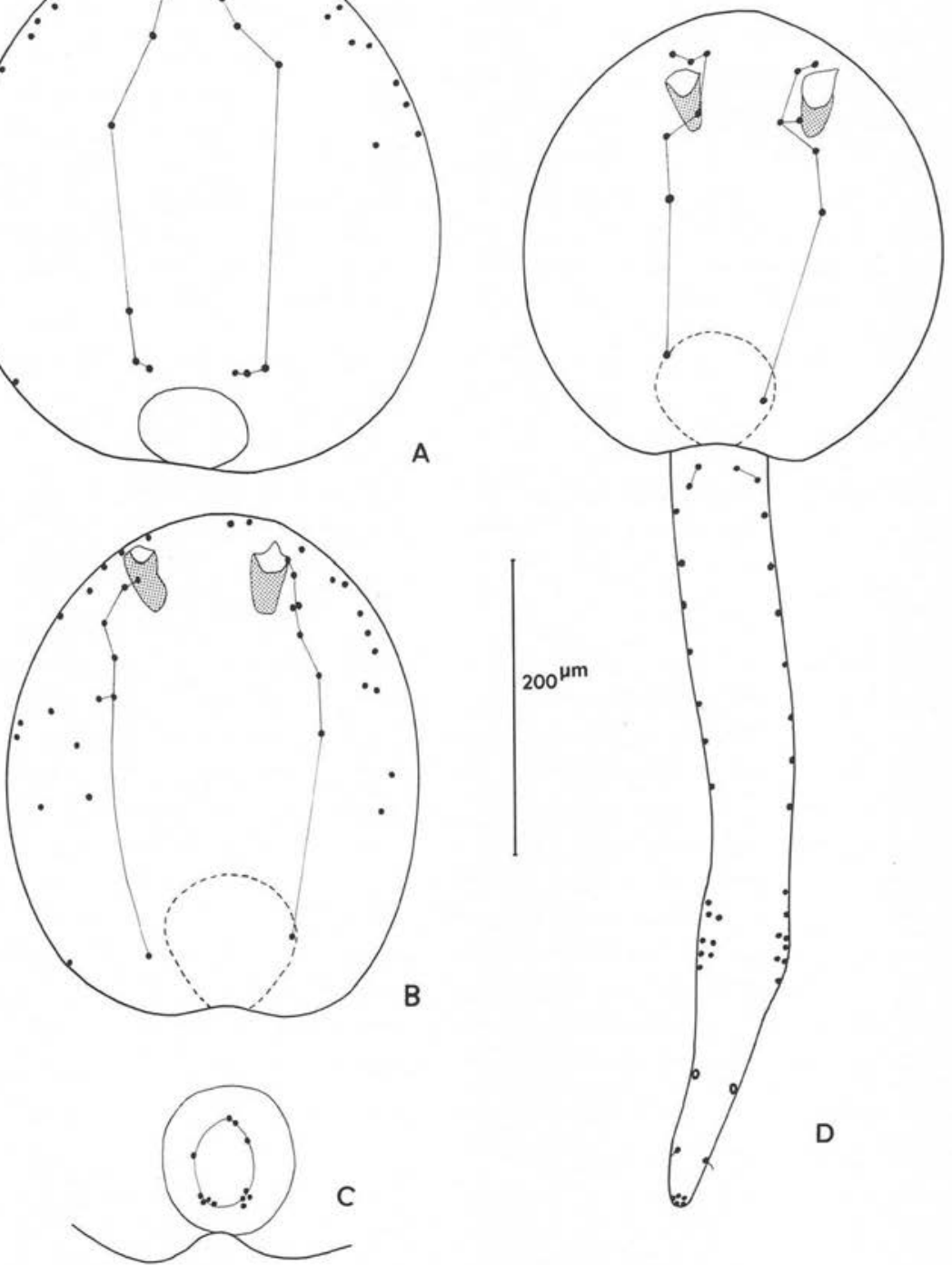

D

C

Fig. 4. Chétotaxie de la cercaire, corps. A : Vue ventrale; B : Vue dorsale; C : Détail de l'acétabulum; D : Vue dorsale, papilles caudales. 
2. Papilles latérales.

$\mathrm{Au}$ nombre d'environ une quinzaine réparties entre les niveaux $\mathrm{AI}$ et $\mathrm{M}$ (fig. $4, A$ et $B$ ).

III. Acetabulum. (fig. 4, C).

Au total $\mathrm{S}=12$ SI.

IV. Queue.

La queue porte au total 16 à 21 paires de papilles et cinq papilles situées à son extrémité postérieure (fig. $4, D$ ).

La disposition des papilles est la suivante :

La première paire est dorsale.

La deuxième paire est dorso-latérale.

A la suite se situent trois groupes de papilles latérales : le premier comprend 7 à 10 paires de papilles latérales assez espacées; le second 6 à 8 paires de papilles latérales serrées disposées en une à deux rangées longitudinales sur un élargissement de la queue ; le troisième groupe comprend une seule paire de papilles latérales situées en arrière des pores excréteurs.

L'extrémité de la queue porte une couronne de cinq papilles.

\section{La métacercaire.}

Les cercaires commencent à s'enkyster environ une demi-heure après leur émission. Les métacercaires sont identiques à celles des autres espèces du genre.

\section{L'adulte.}

Sur le vivant le Ver a une couleur générale rosée qui devient rouge au niveau du pharynx et de l'acétabulum.

Le corps faiblement aplati dorso-ventralement a une forme pyramidale et subit une courbure assez peu marquée qui entraîne une concavité de la face ventrale.

Le tégument est légèrement strié transversalement. D’après les mensurations effectuées sur les coupes sagittales la longueur des vers varie de $6,26 \mathrm{~mm}$ à $7,10 \mathrm{~mm}$. L'acétabulum subterminal est postéro-ventral. Le pore génital bien visible s'ouvre ventralement à la limite du quart antérieur (fig. 5, A).

L'acétabulum a un diamètre externe variant de $1,7 \mathrm{~mm}$ à $2 \mathrm{~mm}$ soit un rapport avec la longueur du corps de $1 / 3,17$ à $1 / 3,90$. Il correspond au type Paramphistomum défini par Nasmark (1937), caractérisé notamment par la présence de deux ensembles distincts de muscles circulaires dorsaux externes nettement séparés (fig. $5, B$ ).

Le nombre des unités pour les différents ensembles musculaires est donné dans le tableau suivant pour cinq spécimens :

\begin{tabular}{|c|c|c|c|c|c|}
\hline Spécimens & De 1 & De 2 & Di & $\mathrm{Vi}$ & $\mathrm{Ve}$ \\
\hline$A, \ldots \ldots \ldots$. & 15 & 16 & 42 & 43 & 19 \\
\hline$\ldots \ldots \ldots \ldots$ & 15 & 17 & 42 & 48 & 17 \\
\hline ........... & 14 & 23 & 47 & 47 & \\
\hline D $\ldots \ldots \ldots$ & 17 & 23 & 40 & 52 & 0 \\
\hline $\mathrm{E} \quad \ldots \ldots \ldots \ldots$ & 15 & 17 & 45 & 45 & 4 \\
\hline
\end{tabular}



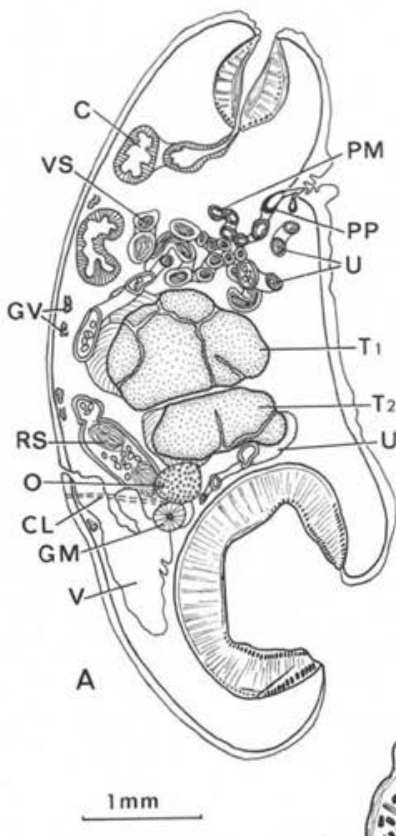
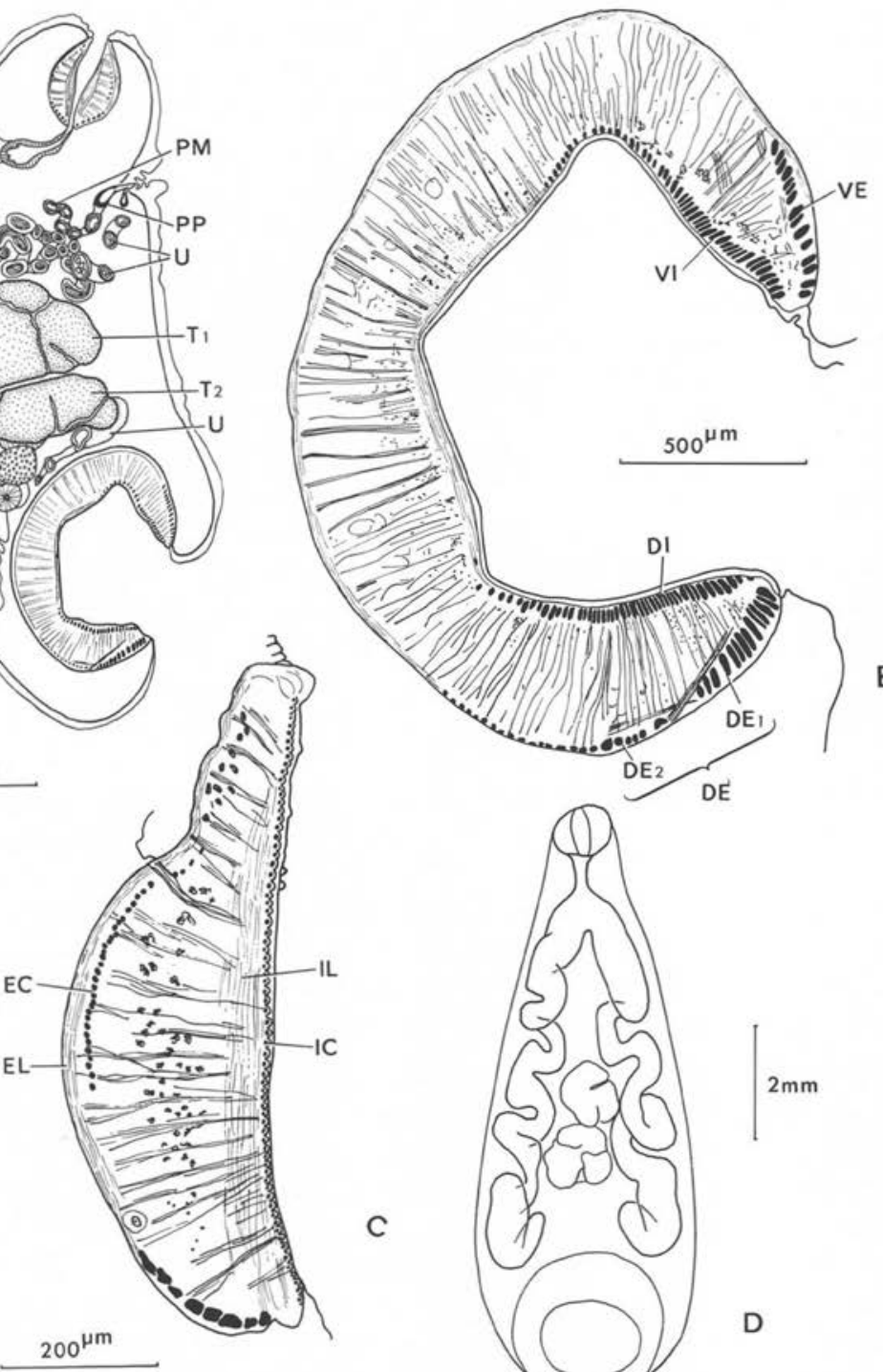

C

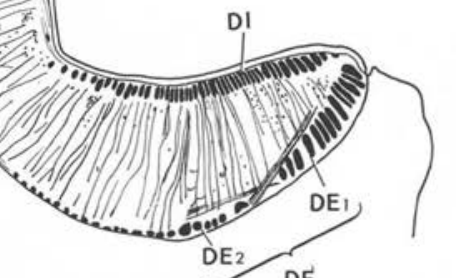

B

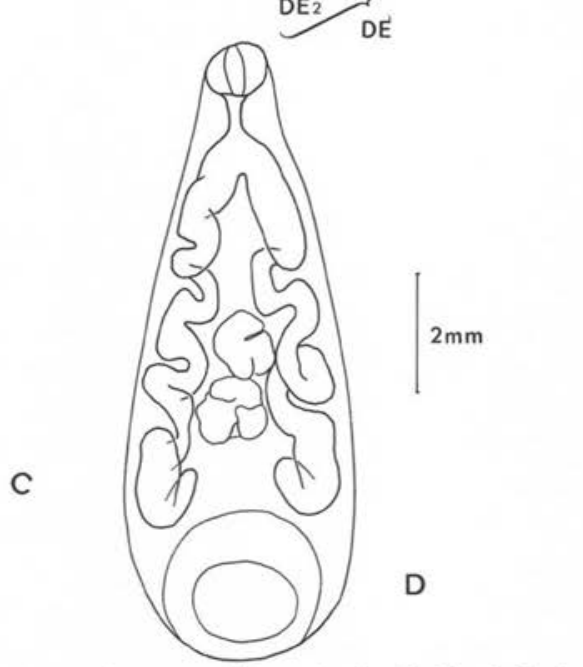

Fig. 5. Adulte. A : Coupe sagittale épaisse; B : Coupe médio-sagittale de l'acétabulum ; C : Coupe médio-sagittale du pharynx; D : Vue ventrale, disposition des caecums.

APPAREIL DIGESTIF.

Le pharynx est long de 740 à $960 \mu \mathrm{m}$ soit un rapport avec la longueur du corps de $1 / 6,70$ à $1 / 8,45$. Son diamètre varie de 640 à $820 \mu \mathrm{m}$. L'absence de sacs et de bulbes pharyngiens, de sphincters et de muscles circulaires médians le rattachent au type Paramphistomum de Nasmark. 
Les muscles circulaires internes sont moyennement développés et s'étendent sur presque toute la longueur du pharynx (fig. 5, C).

Les muscles circulaires externes sont réduits et présents au niveau du $1 / 3$ moyen. Les muscles circulaires basaux sont bien marqués et comportent une dizaine d'unités disposées en une seule couche.

Les muscles longitudinaux internes ont une épaisseur maximum de $50 \mu \mathrm{m}$. Les muscles longitudinaux externes mesurent 15 à $20 \mu \mathrm{m}$ d'épaisseur.

L'œsophage plus court que le pharynx a une direction oblique dorsale. Les deux caecums bien développés subissent un nombre égal de courbures de chaque côté du corps. Leurs extrémités voisines de l'acétabulum ont une direction dorsale et ne se croisent pas (fig. 5, D).

\section{APPAREIL EXCRÉTEUR.}

La vessie, allongée, de contour irrégulier est située dorsalement en arrière de l'acétabulum et du testicule postérieur. Le pore excréteur dorsal s'ouvre à son extrémité antérieure aux $3 / 5$ environ de la longueur du corps (fig. 5, A).

\section{Appareil génital.}

Appareil mâle. Les deux testicules sensiblement identiques, profondément lobés, disposés en tandem, occupent le $1 / 3$ moyen du corps et mesurent environ $1 \mathrm{~mm}$ à $1,2 \mathrm{~mm}$ selon l'axe antéro-postérieur et 1,5 à $1,9 \mathrm{~mm}$ de large.

La vésicule séminale est bien développée et effectue de nombreuses circonvolutions très serrées. La pars musculosa réduite ne forme que deux à trois circonvolutions. Un étranglement la sépare de la pars prostatica longue de 160 à $200 \mu \mathrm{m}$ et large de $120 \mu \mathrm{m}$ qui se prolonge par un court canal éjaculateur (fig. 5, A).

Appareil femelle. L'ovaire de contour subcirculaire en vue sagittale mesure $\mathbf{5 0 0}$ à $600 \mu \mathrm{m}$ de diamètre. Aplati latéralement il est situé entre le testicule postérieur et l'acétabulum dans la moitié gauche du corps (fig. 5, A).

La glande de Melhis sub-sphérique d'un diamètre d'environ $350 \mu \mathrm{m}$ est accolée à l'ovaire en arrière de celui-ci.

Le canal de Laurer, qui en part, débouche dorsalement à $200 \mu \mathrm{m}$ au-dessous du pore excréteur après avoir croisé la vessie.

L'utérus se dirige tout d'abord ventralement au-dessus de l'acétabulum. Il se courbe ensuite, acquiert une direction dorsale puis subit une nouvelle courbure au niveau de laquelle il forme un réceptacle séminal volumineux; il remonte alors vers la région antérieure du Ver en longeant la face dorsale de celui-ci en arrière des testicules. Il se courbe de nouveau au-dessus du testicule antérieur et le métraterme musculeux débouche au pore génital ventral au-dessous du canal éjaculateur.

Les glandes vitellogènes volumineuses constituées de très nombreux follicules s'étendent depuis l'œsophage jusqu'à la moitié de l'acétabulum. Latéralement elles présentent un développement plus important sur la face dorsale du Ver (fig. 6, A). 

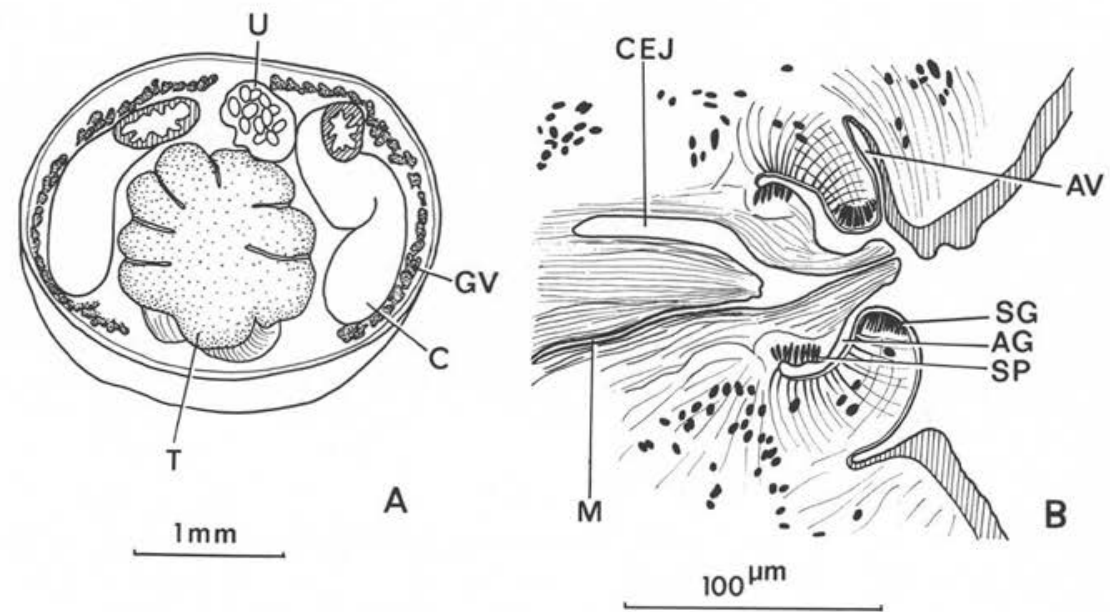

Fig. 6. Adulte. A : Coupe transversale épaisse; B : Pore génital, coupe médio-sagittale.

Le pore génital qui s'ouvre légèrement au-dessous du niveau de la bifurcation des caecums est caractérisé par l'absence d'une ventouse génitale, la présence d'un sphincter génital et d'un sphincter papillaire, de fibres musculaires radiales et d'un atrium ventral réduit. Il appartient donc au type Microbothrium de Nasmark. Le diamètre de l'atrium génital est voisin de $70 \mu \mathrm{m}$, celui de l'atrium ventral varie de 110 à $130 \mu \mathrm{m}$ (fig. 6, B).

\section{Discussion}

Par l'ensemble de ses caractères la forme adulte de l'espèce décrite se rattache au genre Paramphistomum.

Parmi les nombreuses espèces décrites nous retiendrons, en vue de les comparer celles qui présentent comme notre Trématode un acétabulum et un pharynx de type Paramphistomum et un pore génital de type Microbothrium. Ces espèces sont au nombre de deux :

P. vangrembergeni Van Strydonck, 1970.

P. microbothrium Fischoeder, 1901.

P. vangrembergeni Van Strydonck, 1970, parasite de Bovidés et de Cobus sp. au Zaïre, se distingue de l'espèce étudiée par la juxtaposition très accentuée des unités musculaires de l'ensemble De 1 de l'acétabulum, la disposition sur deux rangées des unités musculaires basales du pharynx et la présence au niveau du pore génital d'un sphincter papillaire indistinct.

Les formes larvaires et le Mollusque vecteur de ce parasite ne sont pas connus.

P. microbothrium Fischoeder, 1901, redécrit par Nasmark (1937). Ce parasite et ses formes larvaires ont fait l'objet des travaux de plusieurs auteurs. Il a été observé ou signalé chez des hôtes divers, Gazella dorcas, Bos taurus, Bubalus, Ade- 
nota, Hippotragus, Cobus, Taurotragus sur le continent africain (Dinnik et Dinnik, 1954 ; Swart, 1954), à Madagascar (Prodhon et coll., 1968), en Israël (Lengy, 1960), en Iran (Arfaa, 1962), en Sardaigne (Reinhardt, 1969). P. microbothrium diffère de notre Trématode par le nombre d'unités constituant les différents ensembles de muscles circulaires de l'acétabulum. Toutefois il faut remarquer que sur ce point Nasmark (1937, p. 309 et p. 325) donne deux types de dispositions et que les données des auteurs présentent également des divergences parfois importantes, en particulier pour les ensembles De 1 et De 2 (Swart, 1954 ; Lengy, 1960 (1), Reinhardt, 1969) $P$. microbothrium diffère également de l'espèce décrite par le diamètre nettement supérieur de l'atrium génital et de l'atrium ventral, et le développement de la pars musculosa.

En ce qui concerne les formes larvaires :

Le nombre et la disposition des organites argyrophiles superficiels du miracidium de $P$. microbothrium sont identiques à ceux du miracidium de notre Trématode (Swart, 1967). Par contre le sporocyste est très différent, il a une forme en V fermé chez l'espèce décrite alors que chez $P$. microbothrium il est allongé et présente des parties renflées séparées par des contrictions accusées (Dinnik et Dinnik, 1954). La morphologie générale de la cercaire ne peut guère nous fournir d'éléments de comparaison. Pour ce qui est de la chétotaxie seul Lengy (1960) donne une description partielle des papilles ciliées de la cercaire de $P$. microbothrium mais celle-ci ne nous permet pas une comparaison valable sinon en ce qui concerne l'extrémité de la queue qui porte quatre papilles ciliées (observation faite sur 50 cercaires) alors que chez notre cercaire ces papilles sont de façon constante au nombre de 5 .

Enfin rappelons que le Mollusque vecteur de l'espèce étudiée : B. (B.)forskalii n'a jamais été signalé comme hébergeant des cercaires de $P$. microbothrium et que des essais d'infestation expérimentale de B. (B.) forskalii avec des miracidiums de $P$. microbothrium se sont avérés négatifs (Dinnik, 1965). Ce fait pourrait constituer un argument supplémentaire pour estimer que nous avons affaire à deux espèces distinctes (2).

Nous estimons par conséquent que l'espèce décrite ne pouvant être assimilée à aucune espèce connue est nouvelle et nous la nommons Paramphistomum togolense.

(1) Pour Lengy (1960) le nombre d'unités de l'ensemble De2 varie dans de grandes proportions (3 à 30) sur des coupes parasagittales. Un examen de notre matériel ne met en évidence qu'une légère diminution de ces éléments $(23$ à 18) lorsqu'on s'écarte de façon notable du plan médiosagittal.

(2) A ce propos il faut remarquer que B. (B.) forskalii est également l'hôte intermédiaire naturel et expérimental de Paramphistomum phillerouxi Dinnik, 1961. Cependant, bien que la forme adulte de cette espèce s'apparente à celle de notre Trématode par le type de l'acétabulum et du pore génital, le diamètre très voisin de l'atrium génital et de l'atrium ventral et l'importance réduite de la pars musculosa; elle s'en distingue par la structure du pharynx (présence de $\mathrm{Mcl}$ et d'un sphincter postérieur) et la forme très différente du sporocyste. 


\section{Diagnose}

- Adulte : Longueur : 6,26 à 7,10 $\mathrm{mm}$. Diamètre de l'acétabulum: 1,7 à $2 \mathrm{~mm}$ soit un rapport avec la longueur du corps de $1 / 3,17$ à $1 / 3,90$. Longueur du pharynx : 740 à $960 \mu \mathrm{m}$ soit un rapport avec la longueur du corps de $1 / 6,70$ à $1 / 8,45$.

L'acétabulum, le pharynx et le pore génital sont de type Microbothrium. La pars musculosa est réduite, la vésicule séminale bien développée.

- Sporocyste. Forme caractéristique en V.

Mollusque vecteur, naturel et expérimental : Bulinus (Bulinus) forskalii. Hôte définitif : naturel : inconnu (vraisemblablement un Bovin). Hôte expérimental : Mouton. Localisation : panse.

Origine géographique : Lomé (Togo).

\section{Abréviations UTILISÉES DANS LES Figures,}

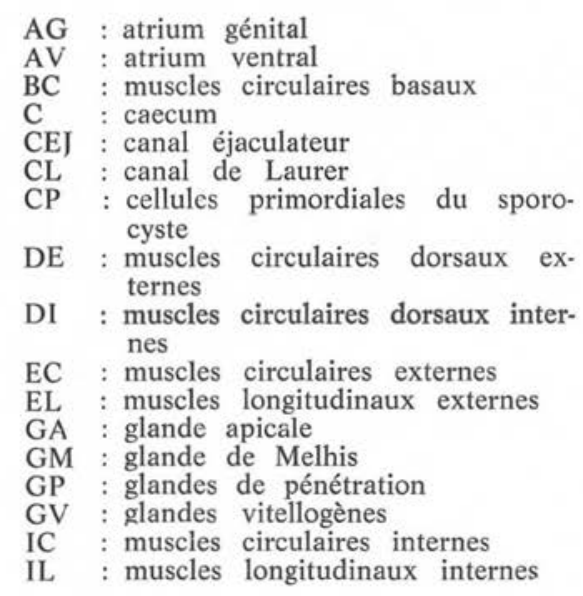

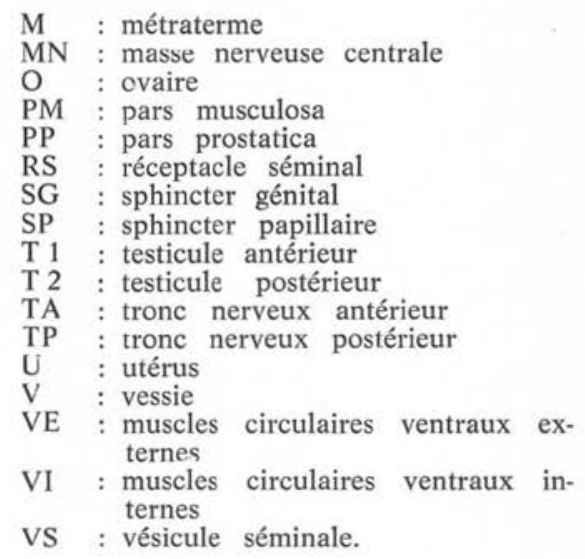

\section{Bibliographie}

Arfaa F. (1962) : A study on Paramphistomum microbothrium in Khuzistan. S. W. Iran. Ann. Parasitol. hum. comp., 37, 549-555.

Chatton E., Lwoff A. (1930): Imprégnation par diffusion argentique de l'infraciliature des Ciliés marins et d'eau douce après fixation cytologique et sans dessication. C.R. Soc. Biol., 104, 834-836.

Combes C., Bayssade-Dufour C., Cassone J. (1976): Sur l'imprégnation et le montage des cercaires pour l'étude chétotaxique. Ann. Parasitol. hum. comp., 51, 399-400.

Dinnik J.A. (1961): Paramphistomum phillerouxi sp. nov. (Trematoda: Paramphistomatidae) and its development in Bulinus forskalii. J. Helminth. 35, 69-90. 
Dinnik J.A. (1965): The snail hosts of certain Paramphistomidae and Gastrothylacidae, discovered by the late $\mathrm{D}^{\mathrm{r}}$ Le Roux in Africa. J. Helminth., 39, 141-150.

Dinnik J.A., Dinnik N.N. (1954): The life cycle of Paramphistomum microbothrium Fischoeder, 1901. Parasitology, 44, 285-299.

Dragesco J., Njine T. (1971): Compléments à la connaissance des Ciliés libres du Cameroun. Ann. Fac. Sci. Yaoundé, (7-8), 97-140.

Fischoeder F. (1901): Die Paramphistomiden der Säugethiere. Zool. Anz., 24, 365-375.

Fischoeder F. (1903): Die Paramphistomiden der Säugethiere. Zool. Jahrb, 17, 485-660.

Lengy J. (1960): Study on Paramphistomum microbothrium Fischoeder, 1901, a rumen parasite of cattle in Israel. Bull. Res. Counc. Isr., Sec. B, Zool. 9B, 71-130.

Lynch J.E. (1933) : The miracidium of Heronimus chelydrae Mac Callum. Quart J. Microsc. Sci., 76, 13-33.

Nasmark K. E. (1937): A revision of the trematode family Paramphistomidae. Zool. Bidr. Uppsala, 16, 301-565.

Prod'hon J., Richard J., Brygoo E. R., Daynes P. (1968): Présence de Paramphistomum microbothrium Fischoeder, 1901, à Madagascar. Arch. Inst. Pasteur Madagascar, 37, 27-30.

Reinhardt S. (1969): Zur Entwicklung, Anatomie und Histologie von Paramphistomum microbothrium (Trematoda, Digenea). Inaug. Dissert. Bonn, 104 p.

Swart P. J. (1954): The identity of so-called Paramphistomum cervi and P. explanatum, two common species of ruminant Trematodes in South-Africa. Onderstepoort. J. Vet. Res., 26, 463-473,

Swart P. J. (1967) : A study of the epidermal structures of the miracidia of Calicophoron calicophorum (Fischoeder, 1901). Näsmark, 1937 and Paramphistomum microbothrium (Fischoeder, 1901). Ibid., 34, 129-136.

Swart P. J., Reinecke R. K. (1962) : Studies on Paramphistomiasis. I. The propagation of Bulinus tropicus Krauss, 1848. Ibid., 29, 183-187.

Van Strydonck D. (1970): Contribution à l'étude de l'anatomie, de la morphologie et de la systématique des Paramphistomidae africains (Plathelminthes: Trematoda). Ann. Mus. Roy. Afr. Centr. Tervuren, Sér. $8^{\circ}$, Sci. zool., 183, 1-56. 